\title{
¿Cómo evolucionan las personas con dependencia de la cocaína que están en tratamiento? Estudio a los tres y seis meses
}

\author{
Ana López Durán (1); Elisardo Becoña Iglesias (1); José Ma García Janeiro (2); Avelina Senra Comesaña(2);

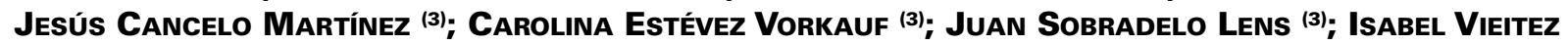 \\ Fernández (3Y 4); Manuel Lloves Moratinos ${ }^{(4)}$; Andrés Moneo Montes ${ }^{(4) ;}$ Lorena Casete Fernández ${ }^{\text {(5y }}$ 6); \\ $M^{a}$ Teresa lage López ${ }^{(5)}$ Y Evaristo Díaz Castro ${ }^{(5)}$.
(1)Departamento de Psicología Clínica y Psicobiología. Facultad de Psicología. Universidad de Santiago de Compostela. (2) Unidad Asistencial del Ayuntamiento de Pontevedra. ${ }^{(3)}$ Unidad Asistencial Alborada. Vigo (Pontevedra).

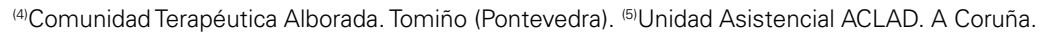 ${ }^{(6)}$ Comunidad Terapéutica ACLAD. A Coruña.
Enviar correspondencia a:
Ana López Durán. Universidad de Santiago de Compostela. Departamento de Psicología Clínica y Psicobiología. \\ Campus Universitario Sur. 15782. Santiago de Compostela. E-mail: alduran@usc.es
}

Recibido: febrero de 2006. Aceptado: septiembre de 2006 .

\section{RESUMEN}

Las demandas de tratamiento en los Centros de Drogodependencias por problemas con el consumo de cocaína se están incrementando en España. El objetivo del presente estudio es en primer lugar, analizar la evolución (a partir de las evaluaciones realizadas por su psicólogo) en las áreas de psicopatología, funcionamiento familiar, laboral y judicial; en segundo lugar, la abstinencia en el consumo de cocaína y en tercer lugar, la permanencia en tratamiento a los tres y seis meses de personas con dependencia de la cocaína en tratamiento en Centros de Drogodependencias de Galicia. En la evaluación inicial disponemos de las evaluaciones realizadas por los psicólogos de 113 sujetos, a los tres meses tenemos datos de 105 sujetos y a los seis de 89 sujetos. A los tres meses, el $58.5 \%$ de los sujetos de los que tenemos datos permanecen abstinentes; a los seis meses siguen sin consumir el 38\%. La evolución en todas las áreas a partir de la evaluación que hace el psicólogo es positiva, encontrándose diferencias significativas en las áreas psicopatológica y familiar. Estos resultados son positivos tanto a nivel de abstinencia, como de retención en tratamiento y mejora en las áreas evaluadas.

Palabras clave: Cocaína, abstinencia, dependencia, tratamiento, seguimiento.

\begin{abstract}
The treatment demands in the Centers of Drug Abuse due to problems with cocaine consumption are increasing in Spain. The objective of the present study is to analyze, firstly the evolution (starting from the evaluations carried out by therapists) in the psychopathological, familiar, labour and judicial areas; secondly, the abstinence in terms of cocaine consumption; and finally, the permanence in treatment of people with cocaine dependence in Galicia (Spain) after three and six months. With regard to initial evolution, we have to our disposal the evaluations carried out by therapists of 113 subjects. After a period of three months, we have data of 105 subjects and after a six-months period we have data of 98 subjects. After the three-months period, the $58.5 \%$ of those previously mensured subjects remain abstinent, and after six months a $38 \%$ continues without consuming cocaine. The evolution in all the areas, starting from the evaluation made by therapits, is possitive and there are even significative difference in the psychopathological and familiar areas. These results are good in terms of abstinence, and retention in treatment and they improve in the evaluated areas.
\end{abstract}

Key words: Cocaine, abstinence, dependence, treatment, follow up.

\section{INTRODUCCIÓN}

- tratamiento de los problemas derivados del consumo de cocaína en los Centros de Drogodependencias españoles es un tema novedoso y relevante en estos momentos. Las demandas de trata- miento por problemas con la cocaína han experimentado un significativo aumento desde el año 1991. En 1991 había 943 personas admitidas a tratamiento por cocaína, en 2002 ascendió a 11.904 (para 7.125 personas era el primer tratamiento por problemas con el 
consumo de drogas). Si analizamos sólo las demandas de tratamientos realizadas por personas que demandan por primera vez tratamiento, la cocaína fue la droga que causó un mayor número de admisiones (41.7\% frente a $28.3 \%$ de heroína) (PNSD, 2005). Este incremento es la consecuencia de la expansión del consumo de cocaína que empezó en España a partir de la segunda mitad de la década de los ochenta, por lo que al aumentar el consumo, las demandas de tratamiento también incrementarán (Galindo 2000, Sanz y Arranz, 2001).

En la encuesta sobre drogas a la población escolar del Plan Nacional sobre Drogas realizada en el año 2004 se ha encontrado un notable incremento del consumo de cocaína de los jóvenes escolarizados entre 14 y 18 años de edad. En 1994 un 2,4\% habían consumido alguna vez cocaína y un 1,7\% lo habían hecho en los últimos doce meses; en 2000 un 5,4\% había consumido cocaína alguna vez y un $4 \%$ lo había hecho en los últimos doce meses, y un 2,2\% en los últimos 30 días; en 2002 un 7,4\% ha consumido cocaína alguna vez en la vida, un $6 \%$ en los últimos doce meses y un $3,1 \%$ en los últimos 30 días; en 2004 un 8,5\% había consumido alguna vez en la vida cocaína, un 6,8\% en el último año, y un 3,6\% en el último mes (PNSD, 2004b). Estos datos nos indican que el consumo de cocaína en jóvenes se ha multiplicado por dos o tres en estos últimos diez años. En el estudio realizado en 2002 (PNSD, 2004c) la media de edad de inicio en el consumo es de 15,7 años (no hay diferencia con los estudios previos en cuanto a la edad de inicio).

En las encuestas domiciliarias sobre consumo de drogas que realiza el Plan Nacional sobre Drogas en la población general, la última en el año 2003 (PNSD, 2005), se observa también un incremento en el consumo de cocaína. En los estudios realizados en 1995, 1997 y 1999 , el porcentaje de personas que consumieron cocaína alguna vez en la vida es algo superior al $3 \%$ en los tres casos; los consumos en el último mes también permanecen estables, en 1997 y en 1999 un 0,9\% de la población había consumido cocaína en el último mes (PNSD, 2004a). Pero en la encuesta domiciliaria realizada durante el año 2001, ya se observa un fuerte incremento en el consumo: el consumo alguna vez en la vida es de 4,8\%; en el último año es de $2,5 \%$ y en el último mes es de 1,1\%. Este incremento se sigue manifestando en la última encuesta domiciliaria, la del año 2003: un 5,9\% de la población española entre 15 y 64 años ha consumido cocaína alguna vez en la vida, y un $2,7 \%$ lo ha hecho en el último año. En cambio, el consumo en el último mes ha disminuido respecto a la encuesta anterior, un 1,3\% en el 2001 frente a un 1,1\% en el 2003. La edad media de inicio del consumo en el año 2003 ha disminuido respecto a los datos de 1999 en los que se situaba en 21,6 años. En el último estudio realizado en 2003 la edad media de inicio en el consumo de cocaína es de 20,9 años (PNSD, 2004a).

Por lo tanto, los estudios que analizan aspectos relacionados con el consumo de cocaína son muy relevantes en estos momentos. Pero todavía siguen siendo pocos los estudios realizados en nuestro país con personas con dependencia de la cocaína que están a tratamiento en Centros de Drogodependencias. Quizás porque es un fenómeno reciente los estudios de seguimiento son todavía escasos (Pedrero, Puerta, Segura y Martínez, 2004; Sánchez-Hervás, Morales, Tomás, Molina, del Olmo, Bosch, Sanoguera y Escrivá, 2002).

A nivel internacional, en relación a estudios de pacientes tratados y a su evolución posterior, destaca el realizado por Carroll, Power, Bryant y Rounsaville (1993). En él participaron 298 sujetos aunque sólo pudieron ser evaluados 98 al año de inicio del tratamiento. Estos autores señalaron que tras un año de tratamiento un tercio de la muestra se ha mantenido abstinente, pero la existencia de abstinencia no significa que haya mejoría en todos los ámbitos. McKay, Merikle, Mulvaney, Weiss y Koppenhaver (2001) realizaron un estudio de seguimiento con 132 veteranos de guerra con diagnóstico de dependencia de la cocaína alguna vez en la vida. A los seis meses del inicio del tratamiento el porcentaje de sujetos que habían vuelto a consumir cocaína era del $47 \%$.

Pero el estudio más amplio realizado con personas con problemas con el consumo de cocaína es el de Crist-Christoph et al. (1999). Estos autores realizaron un estudio con 487 sujetos consumidores de cocaína para comparar cuatro tipos de tratamientos. El porcentaje de abstinencia a los tres meses de iniciados los tratamientos era de $26.4 \%$.

El objetivo del presente estudio es analizar la evolución del tratamiento (abstinencia o consumo) y el funcionamiento de personas con dependencia de la cocaína a partir de las evaluaciones que realiza el psicólogo de referencia en tres momentos diferentes: cuando se realiza la evaluación psicológica del sujeto, a los tres y a los seis meses de la misma. Los ámbitos que se evalúan son: psicopatología, área social/familiar, área laboral/académica y área judicial. A los tres y a los seis meses se evalúa la abstinencia y si el paciente mantiene el contacto con el Centro de Drogodependencias.

\section{MÉTODO}

\section{Participantes}

Durante la realización del estudio fueron evaluados inicialmente 119 sujetos que solicitaron tratamiento en cinco centros públicos de tratamiento de Drogodepen- 
dencias de Galicia (Unidad Asistencial del Concello de Pontevedra, Unidad Asistencial de ACLAD Alborada (Vigo, Pontevedra), Comunidad Terapéutica de ACLAD Alborada (Tomiño, Pontevedra), Unidad Asistencial de ACLAD (A Coruña) y Comunidad Terapéutica de ACLAD (A Coruña). De estos sujetos fueron eliminados cuatro por diversas razones: un sujeto no cumplía los criterios para el diagnóstico de dependencia de la cocaína evaluados con la SCID-I (First, Spitzer, Gibbson y Williams, 1998), y tres sujetos superaban las seis semanas de abstinencia. Realizamos la evaluación inicial a 115 sujetos que cumplían los criterios de inclusión en el estudio pero sólo disponemos de las evaluaciones realizadas por los psicólogos de los Centros de 113 de ellos en el momento de la evaluación inicial. A los tres meses tenemos la evaluación completa realizada por los psicólogos de 105 sujetos y a los seis meses de 89 sujetos.

También analizamos la continuidad en el tratamiento en el recurso asistencial. A los tres meses disponemos de información de 108 sujetos, ya que 5 son considerados perdidos porque no volvieron al centro tras realizar la evaluación inicial. A los seis meses tenemos información de 96 sujetos, 17 son considerados perdidos. Cinco no volvieron al centro tras la evaluación inicial y 15 no volvieron al centro tras la evaluación de tres meses.

Respecto a la evaluación del mantenimiento de la abstinencia a partir de las analíticas de orina, disponemos a los tres meses de la información de 99 sujetos y a los seis meses, de 91 sujetos.

Estas diferencias en el tamaño de la muestra están causadas por diversas razones: en algunos casos disponemos de la evaluación del psicólogo del Centro pero no hay analíticas de orina para confirmar la abstinencia, en otros casos el sujeto hace los controles de orina pero ha dejado de acudir al psicólogo por lo que conocemos si consume o no pero no su evolución, y en la mayoría de los casos en los que el sujeto es derivado a otro recurso asistencial no tenemos información de cómo evoluciona y de si ha consumido o no. A los tres meses disponemos de la información completa (evaluación del psicólogo y analíticas de orina) de 97 sujetos, y a los seis meses de 78.

La muestra fue seleccionada entre el 23 de septiembre de 2003 y el 28 de abril de 2005 en función de los siguientes criterios de inclusión: estar a tratamiento por consumo de cocaína como droga principal, existencia de dependencia de la cocaína según los criterios del DSM-IV-TR (American Psychiatric Association, 2002), y llevar un mínimo de tres semanas y un máximo de seis semanas abstinente del consumo de cocaína. Los criterios de exclusión fueron: la presencia de alteraciones psicóticas graves y la incapacidad de responder a los instrumentos de evaluación utilizados

\section{Instrumentos de evaluación}

Evaluación global del paciente a realizar por el psicólogo de referencia. (ver anexo 1) Cuando se evalúa a un sujeto, su psicólogo debe determinar en una escala tipo Likert de cero a cuatro cómo considera que se encuentra el sujeto en las áreas psicopatológica, familiar/social, laboral y judicial. En el área psicopatológica, cero indica ausencia de síntomas, uno indica presencia de algunos síntomas leves, dos indica presencia de síntomas moderados, tres indica presencia de un trastorno psicopatológico grave y cuatro indica que el paciente presenta una alteración de la realidad o de la comunicación. En el área familiar/social, cero indica que hay un funcionamiento normalizado, uno que presenta algunas dificultades, dos que tiene dificultades moderadas, tres que tiene dificultades importantes en las relaciones y cuatro que hay una alteración grave en el área de las relaciones. En el área laboral, cero indica que hay un funcionamiento normalizado, uno que hay algunas dificultades, dos que hay dificultades moderadas, tres que hay dificultades importantes y cuatro que hay una alteración grave en esta área. En el área judicial, cero indica que no tiene problemas judiciales, uno que está acusado de cometer delitos leves, dos que está acusado de cometer delitos importantes, tres que se encuentra en libertad condicional y cuatro que en la actualidad está cumpliendo condena.

A los tres y seis meses de la evaluación inicial el psicólogo debe cubrir nuevamente este cuestionario e indicar además si el sujeto ha realizado nuevos consumos de cocaína desde la evaluación inicial, y si muestra adherencia al tratamiento. Para determinar la existencia de nuevos consumos y para la validación de la abstinencia se realizaron analíticas de orina a los tres y seis meses de la evaluación inicial en todos los sujetos.

Para la elaboración de este instrumento nos basamos en la Escala de Evaluación de la Actividad Global del DSM-IV - TR (2002). En dicha escala se valora el funcionamiento psicológico, laboral y social (nosotros añadimos el área familiar) basándose en la existencia de un continuum entre salud y enfermedad.

Entrevista Clínica Estructurada para el DSM-IV (SCID) de First et al. (1998) en su versión española, para evaluar la dependencia de la cocaína, que se aplicó en la evaluación inicial. 


\section{Procedimiento}

Los pacientes fueron seleccionados consecutivamente por los psicólogos de los Centros de Drogodependencias, entre septiembre de 2003 y abril de 2005. Si cumplían los criterios de inclusión en el estudio eran citados para ser evaluadas sus características sociodemográficas, aspectos relacionados con el consumo de drogas, diagnóstico de la dependencia y características psicopatológicas. En este momento el psicólogo cubre por primera vez el cuestionario de evaluación global que evalúa las áreas de psicopatología, y de funcionamiento familiar, laboral y judicial. Todos los sujetos que participaron en el estudio firmaron el consentimiento informado.

A los tres y seis meses de realizada esta evaluación el psicólogo de referencia evalúa nuevamente cómo considera que es el funcionamiento del sujeto en las mismas áreas. Además, el psicólogo debe de indicar si el sujeto ha realizado nuevos consumos de cocaína desde la evaluación inicial comprobados mediante analíticas de orina y si mantiene el contacto con el centro. El instrumento para realizar las analíticas de orina en todos los centros que participaron en el estudio es el ETS PLUS SYVA, que realiza análisis de tipo enzimoimnulógico.

\section{Tratamiento}

El tratamiento psicológico aplicado a las personas de esta muestra es el habitual en estos Centros de Tratamiento. La mayor parte de ellos realizan un tratamiento consistente en intervención de tipo cognitivoconductual con prevención de la recaída que es el que tiene evidencia científica de ser eficaz (Crist-Christoph et al., 1999). El tratamiento farmacológico es una variable difícil de controlar en el estudio. Principalmente porque entre las personas con dependencia de sustancias es probable la presencia de otros trastornos psiquiátricos (López y Becoña, 2006) que requieren una medicación determinada. Para abordar el problema de la dependencia de la cocaína no existe un tratamiento farmacológico específico como en el caso de la heroína, por lo que los fármacos que se administran son para abordar determinados aspectos del consumo de cocaína pero no para el tratamiento de la dependencia específicamente. Para el tratamiento de los síntomas derivados del síndrome de abstinencia se utilizan antidepresivos, y para el control de la impulsividad se utiliza el topiramato, preferentemente.

El presente estudio no tenía por objetivo evaluar la eficacia del tratamiento de cada centro, sino la eficacia global del tratamiento en los Centros de Drogodependencias con estos adictos, personas con dependencia de la cocaína, a los tres y seis meses de la evaluación inicial.

\section{Tratamiento estadístico}

Se utilizó el paquete estadístico SPSS para Windows (versión 12.0). Se realizaron análisis descriptivos, de frecuencias, la prueba t para las comparaciones entre grupos y análisis de varianza con medidas repetidas (inicio, tres y seis meses) con el análisis post hoc de Bonferroni.

\section{RESULTADOS}

A nivel descriptivo los principales resultados que hemos obtenido han sido los siguientes. A los tres meses de realizada la evaluación inicial 5 sujetos (4.4\%) son considerados como sujetos perdidos, con los que no se tiene ningún contacto. A los seis meses, 17 sujetos $(15.0 \%)$ son considerados como perdidos. Han sido derivados a otro recurso asistencial a los tres meses 9 sujetos $(7.9 \%)$ y a los seis meses 11 sujetos (9.7\%). A los seis meses habían recibido el alta terapéutica 4 sujetos (3.5\%). Por lo tanto, se mantenía el contacto con 99 sujetos (87.6\%) a los tres meses y con 81 sujetos $(71.6 \%)$ a los seis meses (ver tabla 1$)$.

Tabla 1. ¿Es un caso perdido?

\begin{tabular}{|l|c|c|c|c|}
\hline & \multicolumn{2}{|c|}{ A los 3 meses } & \multicolumn{2}{c|}{ A los 6 meses } \\
& $N$ & $\%$ & \multicolumn{2}{c|}{ \% } \\
\hline Sí & 5 & 4.4 & 17 & 15.0 \\
\hline No & 99 & 87.6 & 81 & 71.6 \\
\hline $\begin{array}{l}\text { Derivado a otro } \\
\text { recurso asistencial }\end{array}$ & 9 & 7.9 & 11 & 9.7 \\
\hline Alta terapéutica & 0 & 0 & 4 & 3.5 \\
\hline
\end{tabular}

Respecto a la abstinencia, a los tres meses de realizada la evaluación inicial 58 sujetos $(50.4 \%)$ no habían vuelto a consumir cocaína, 41 sujetos (35.7\%) habían consumido y había 16 sujetos (13.9\%) de los que desconocemos si han consumido o no. A los seis meses de la evaluación inicial, 35 sujetos (30.4\%) permanecían abstinentes en el consumo de cocaína, 57 sujetos (49.6\%) habían vuelto a consumir y desconocíamos en 23 sujetos (20.0\%) si lo habían hecho. Considerando sólo a los sujetos localizados el porcentaje de abstinencia es del $58.5 \%$ a los tres meses y del $38 \%$ a los seis meses. Señalar que la existencia de un consumo puntual de cocaína en los tres primeros meses tras la realización de la evaluación inicial, ya implica que se clasifique al sujeto en la evaluación a los seis meses dentro del grupo de consumo. El psicólogo indica en cada evaluación si el sujeto ha realizado nuevos consumos desde el momento de la evaluación inicial, por lo tanto estamos evaluando la abstinencia continua a los tres y a los seis meses (ver tabla 2 y figura 1). 
Tabla 2. ¿Ha vuelto a consumir cocaína?

\begin{tabular}{|c|c|c|c|c|}
\hline & A 10 & neses & A lo & heses \\
\hline & $N$ & $\%$ & $N$ & $\%$ \\
\hline Sí & 41 & 35.7 & 57 & 49.6 \\
\hline No & 58 & 50.4 & 35 & 30.4 \\
\hline Desconocido & 16 & 13.9 & 23 & 20.0 \\
\hline
\end{tabular}

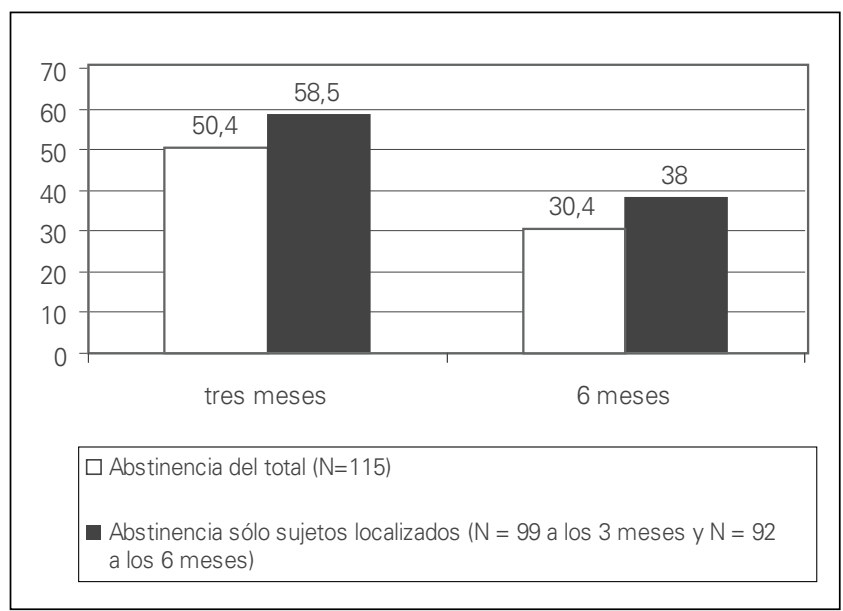

Figura 1. Porcentaje de abstinencia en el consumo de cocaína a los tres y seis meses (abstinencia continua)
Respecto a las puntuaciones medias en los distintos ámbitos que debía de evaluar el psicólogo (en una escala tipo Likert entre cero y cuatro) (ver tabla 3), en el área de psicopatología, en el momento de la evaluación inicial ( $N=113$ ) la puntuación media es de 1.59, a los tres meses es de $1.18(\mathrm{~N}=105)$, y a los seis meses es de 1.21 ( $N=89$ ). En el área del funcionamiento familiar la puntuación media en el momento de la evaluación inicial era de 1.61 ( $N=113$ ), a los tres meses es de $1.18(\mathrm{~N}=105)$ y a los seis meses es de $1.31(\mathrm{~N}=89)$. En el área del funcionamiento laboral la puntuación media en la evaluación inicial es de 1.37 ( $N=108$ ), a los tres meses es de 1.06 ( $N$ =97), y a los seis meses es de $1.22(\mathrm{~N}=85)$. En el área de problemas judiciales la puntuación media en la evaluación inicial es de $0.46(\mathrm{~N}=113)$, a los tres meses es de 0.35 ( $N=105)$ y a los seis meses es de $0.36(\mathrm{~N}=88)$. Como se aprecia, en todas las áreas que son evaluadas por el psicólogo hay una evolución positiva, ya que la puntuación media disminuye respecto a la evaluación inicial. Hay diferencias significativas en el área psicopatológica $\left(F_{(2)}=6.782 ; p<.01\right) \mathrm{y}$ en el área familiar $\left(F_{(2)}=4.895 ; p<.05\right)$. Aplicamos la prueba post hoc de Bonferroni para determinar cuáles son las evaluaciones entre las que hay diferencias significativas. En la evaluación del área psicopatológica hay diferencias significativas entre la evaluación inicial y la evaluación a los tres meses ( $p<.01)$, y entre la evaluación inicial y la evaluación a los seis meses ( $p<$ .01). En la evaluación del área familiar hay diferencias significativas entre la evaluación inicial y la evaluación a los tres meses ( $p<.01)$, y la evaluación inicial y la evaluación a los seis meses $(p<.05)$.

Tabla 3. Puntuaciones medias obtenidas en la escala de evaluación del psicólogo

\begin{tabular}{|l|c|c|c|c|c|c|c|c|c|}
\hline & \multicolumn{3}{|c|}{ Inicial } & \multicolumn{3}{c|}{ Tres meses } & \multicolumn{3}{c|}{ Seis meses } \\
\hline & $N$ & Media & D.T. & $N$ & Media & D.T. & N & Media & D.T. \\
\hline Psicopatología & 113 & 1.59 & 0.89 & 105 & 1.18 & 0.90 & 89 & 1.21 & 0.91 \\
\hline Familiar & 113 & 1.61 & 1.09 & 105 & 1.18 & 1.01 & 89 & 1.31 & 1.27 \\
\hline Laboral & 108 & 1.37 & 1.18 & 97 & 1.06 & 1.13 & 85 & 1.22 & 1.26 \\
\hline Judicial & 113 & 0.46 & 0.91 & 105 & 0.35 & 0.82 & 88 & 0.36 & 0.77 \\
\hline
\end{tabular}

Nota. La escala va de cero (ausencia de síntomas) a cuatro (hay una alteración grave)

Destaca el incremento del porcentaje de sujetos que tienen un funcionamiento normalizado en cada una de las áreas evaluadas a los tres y seis meses. En el área psicopatológica el porcentaje de sujetos que no presenta ningún síntoma de este tipo en el momento de la evaluación inicial es del 9.7\%, asciende al $25.7 \%$ a los 3 meses y es del $25.8 \%$ a los seis meses. En el área familiar/social, presentan un funcionamiento normalizado en el momento de la evaluación inicial el $13.3 \%$ de los sujetos. El porcentaje asciende al 23.8\% a los 3 meses y $30.3 \%$ a los seis meses. En el área laboral/académica, en la evaluación inicial el 27.8\% de 
los sujetos tiene un funcionamiento normalizado, a los tres meses se incrementa el porcentaje al 39.2\%, y a los seis meses se sitúa en el 38.8\%. En el área judicial, hay un $73.5 \%$ de los sujetos que no tienen problemas de este tipo en el momento de la evaluación inicial. Este porcentaje se incrementa al $80.0 \%$ a los tres meses y es del $78.4 \%$ a los seis meses.

Para finalizar, también analizamos si existe relación entre la existencia de nuevos consumos de cocaína y una evolución positiva en las cuatro áreas evaluadas por el psicólogo (psicopatología, familiar, laboral y judicial). Sólo analizamos aquellos sujetos de los que disponíamos la información sobre la existencia de nuevos consumos y la evaluación realizada por el psicólogo (n
$=97$ a los tres meses; $n=78$ a los seis meses). No incluimos los casos en los que tenemos información sobre si realizó nuevos consumos pero no la evaluación del psicólogo (ej. sólo lo ve el médico y ya no lo ve el psicólogo) y los que no sabemos si consumió o no pero el psicólogo cubrió la hoja de evaluación (ej. mantiene contacto telefónico con el paciente). Sólo hay diferencias significativas a los tres meses de la evaluación inicial en el área familiar. Los sujetos que no han realizado nuevos consumos de cocaína a los tres meses de la evaluación, tienen una puntuación media inferior (0.91) en el área familiar que los sujetos que han consumido cocaína $(1.48)\left(t_{(1,196)}=-2.872 ; p\right.$ $<.01$ ) (ver tabla 4).

\section{Tabla 4. Puntuación media en las áreas evaluadas en sujetos que han vuelto a consumir cocaína y sujetos que mantienen la abstinencia.}

\begin{tabular}{|c|c|c|c|c|c|c|c|c|c|c|c|c|c|}
\hline & & \multicolumn{3}{|c|}{ Psicopatológica } & \multicolumn{3}{|c|}{ Familiar } & \multicolumn{3}{|c|}{ Laboral } & \multicolumn{3}{|c|}{ Judicial } \\
\hline & & $N$ & Media & D.T. & $\mathrm{N}$ & Media & D.T. & $N$ & Media & D.T. & $N$ & Media & D.T. \\
\hline \multirow{2}{*}{$\begin{array}{l}\infty \\
\mathbb{1} \\
\infty \\
\Phi \\
E \\
m\end{array}$} & Abstinente & 56 & 1.05 & 0.88 & 56 & 0.91 & 0.92 & 52 & 0.98 & 1.07 & 56 & 0.44 & 0.89 \\
\hline & $\begin{array}{l}\text { No abstinen- } \\
\text { te }\end{array}$ & 41 & 1.36 & 0.82 & 41 & 1.48 & 1.05 & 38 & 1.13 & 1.18 & 41 & 0.26 & 0.77 \\
\hline \multirow{2}{*}{$\begin{array}{l}\mathscr{D} \\
\infty \\
\mathbb{1} \\
\varepsilon \\
0\end{array}$} & Abstinente & 34 & 1.05 & 0.81 & 34 & 1.02 & 1.05 & 33 & 0.87 & 0.89 & 33 & 0.51 & 0.90 \\
\hline & $\begin{array}{l}\text { No abstinen- } \\
\text { te }\end{array}$ & 44 & 1.31 & 0.95 & 44 & 1.45 & 1.35 & 41 & 1.41 & 1.46 & 44 & 0.25 & 0.61 \\
\hline
\end{tabular}

\section{DISCUSIÓN}

En este estudio sobre la evolución a los tres y seis, en personas con dependencia de la cocaína en tratamiento, los resultados señalan que en las cuatro áreas que son evaluadas por los psicólogos a los tres y a los seis meses de la evaluación inicial, área psicopatológica, familiar/social, laboral/académica y judicial, existe una evolución positiva. Por lo tanto, en aquellos sujetos en los que tenemos datos de su evolución a los tres $(n=105)$ y a los seis meses $(n=89)$, se puede considerar la existencia de una evolución positiva en las áreas evaluadas por el psicólogo. Hay diferencias significativas en el área de psicopatología y de funcionamiento familiar, las personas que forman la muestra tienen un mejor funcionamiento en estas áreas a los tres y a los seis meses de la evaluación inicial.

Respecto a la existencia de nuevos consumos de cocaína desde que se hizo la evaluación inicial, el porcentaje de abstinencia entre los sujetos que forman la muestra a los tres meses de la evaluación inicial es superior al $30 \%$ señalado por Crist-Christoph et al. (1999) y por Higgins, Budney, Bickel, Foerg, Donham y Badger (1994). En el presente estudio a los tres meses de la evaluación inicial, el $50.4 \%$ de los sujetos no habían vuelto a consumir cocaína. A los seis meses el porcentaje de sujetos que no han realizado nuevos consumos es del $30.4 \%$ en la muestra disponible, es un porcentaje inferior al $47 \%$ de abstinencia que señalan McKay et al. (2001), aunque debemos tener en 
cuenta que la muestra que utilizan estos autores tiene unas características muy específicas: todos hombres, la mayor parte de raza negra, veteranos de guerra y que están en un programa de tratamiento de cuidados continuos.

Debemos añadir, que tal y como apuntan Carroll et al. (1993) en un estudio longitudinal para analizar como influye la psicopatología y la severidad de la dependencia en los resultados del tratamiento, el mantenimiento de la abstinencia no implica necesariamente la existencia de mejoría en todas las áreas afectadas por el consumo de sustancias. Como señalan nuestros resultados, la única variable relacionada con el mantenimiento de la abstinencia es el funcionamiento en el área familiar a los tres meses de la evaluación inicial. Los sujetos que mantienen la abstinencia tienen un mejor funcionamiento en esta área. Esto coincide con estudios realizados con alcohólicos, como el de Longabaugh, Wirtz, Beattie, Noel y Stout (1995), que apuntan que la existencia de apoyo familiar y social predice un mejor resultado en el tratamiento de los problemas con el alcohol, y más concretamente con el mantenimiento de la abstinencia.

Respecto a la retención en el tratamiento, en el presente estudio a los tres meses continuaban en tratamiento en el mismo recurso asistencia el 87.6\% de los sujetos ( $n=99$ ) y habían sido derivados a otro recurso el $7.9 \%(n=9)$. Este porcentaje de retención es muy superior al señalado en el estudio de Nancy et al. (2005): a los tres meses el $49 \%$ de los sujetos en tratamiento con un programa de incentivos continuaba en tratamiento, frente al $35 \%$ de retención del grupo que no recibía incentivos. También es un porcentaje de retención superior al obtenido en el estudio de Bisaga et al. (2006) para evaluar el tratamiento con gabapentina: el $49 \%$ de la muestra inicial completó el tratamiento de tres meses con este fármaco.

Por lo tanto, a partir de nuestros resultados podemos concluir que los sujetos con dependencia de la cocaína en tratamiento tienen una evolución positiva en todas las áreas evaluadas por los psicólogos a los tres y seis meses de realizada la evaluación inicial. El porcentaje de abstinencia a los tres meses de la evaluación inicial (50.4\%) es muy superior al señalado en el estudio de Crist-Christoph et al. (1999), tal y como lo hemos evaluado con verificaciones de la abstinencia mediante analíticas de orina, de los sujetos evaluados en ese tiempo. A los seis meses de la evaluación inicial el porcentaje de sujetos que se mantienen abstinentes desciende hasta el $30.4 \%$ (38\% si no se consideran los sujetos perdidos). Igualmente, este estudio nos indica que el mantenimiento de la abstinencia no está relacionado con un mejor funcionamiento en todas las áreas evaluadas, excepto en el ámbito familiar a los tres meses. Las personas que se mantienen abstinentes a los tres meses de la evaluación tienen un mejor funcionamiento en el área familiar.

Hay que indicar que los programas de tratamiento en las Unidades Asistenciales de Drogodependencias de España tienen una duración de varios meses. Esto puede explicar en parte los resultados, dado que de toda la muestra sólo un $3.5 \%$ ha sido dada de alta a los seis meses; el resto está todavía en tratamiento (el $71.6 \%$ en el mismo centro y el $9.7 \%$ derivado a otro recurso asistencial a los seis meses) o han dejado de acudir a tratamiento (el $15.0 \%$ a los seis meses). Esto sugiere que retenemos a los pacientes en tratamiento, como ocurre en el presente caso, para facilitar el mantenimiento de la abstinencia en los mismos.

Futuras investigaciones deben de confirmar los resultados obtenidos en este estudio, realizar seguimientos más largos y analizar la eficacia de los tratamientos que se aplican en los Centros de Drogodependencias con seguimientos más largos, tanto si con ellos se sigue aplicando un tratamiento, como si ya han sido dados de alta.

\section{REFERENCIAS}

American Psychiatric Association (2002). DSM-IV-TR. Manual diagnóstico y estadístico de los trastornos mentales. Barcelona: Masson (Original 2000).

Bisaga, A., Aharonovich, E., Garawi, F., Levin, F. R., Rubin, E., Raby, W. N. y Nunes, E. V. (2006). A randomized placebo-controlled trial of gabapentin for cocaine dependence. Drug Alcohol Depend, 81, 267-274.

Carroll, K.M., Power, M.D., Bryant, K. y Rounsaville, B.J. (1993). One-year follow-up status of treatment-seeking cocaine abusers. J Nerv Ment Dis, 181, 71-79.

Crist-Christoph, P., Siqueland, L., Blaine, J., Frank, A., Luborsky, L., Onken, L.S., Muenz, L.R, Thase, M.E., Weiss, R.D., Gastfirnd, D.R., Woody, G.E., Barber, J.P., Butler, S.F., Daley, D., Salloum, I., Bishop, S., Najavits, L.M., Lis, J., Mercer, D., Griffin, M.L., Moras, K. y Beck, A.T. (1999). Psychosocial treatments for cocaine dependence. National Institute on Drug Abuse Colloborative Cocaine Treatment Study. Arch Gen Psychiatry, 56, 493-501.

First, M.B., Spitzer, R.L., Gibbson, M., Williams, J.B.W. y Smith-Benjamin, L. (1998). Guía del usuario de la entrevista clínica estructurada para los trastornos del eje I del DSM-IV- SCID-I. Barcelona: Masson.

Galindo, A. (2000). Manejo del paciente con dependencia a la cocaína. Trastornos Adictivos, 2, 122-132.

Higgins, S.T., Budney, A. J., Bickel, W. K., Foerg, F. E., Donham, R. y Badger, G.J. (1994). Incentives improve 
outcome in outpatient behavioral treatment of cocaine dependence. Arch Gen Psychiatry, 51, 568-576.

Longabaugh, R., Wirtz, P.W., Beattie, M.C., Noel, N. y Scout, R.L. (1995). Matching treatment focus to patient social investiment and support: 18 month follow-results. J Consult Clin Psychol, 63, 296-307.

López, A. y Becoña, E. (2006). Consumo de cocaína y psicopatología asociada: una revisión. Adicciones, 18, en prensa.

Mckay, J.R., Merikle, E., Mulvaney, F.D., Weiss, R.V. y Koppenhaver, J.M. (2001).

Factors accounting for cocaine use two years following initiation of continuing care. Addiction, 96, 213-225.

Nancy, M. P., Peirce, J. M., Stitzer, M. L., Blaine, J., Roll, J. M., Cohen, A. et al. (2005). Effect of prize-based incentives on outcomes in stimulant abusers in outpatient psychosocial treatment programs. Arch Gen Psychiatry, 62, 1148-1156.

Pedrero, E.J., Puerta, C., Segura, I. y Martínez, S. (2004). Evolución de la sintomatología psicopatológica de los drogodependientes a lo largo del tratamiento. Trastornos Adictivos, 6, 175-191.
Plan Nacional sobre Drogas (2005). Observatorio Español sobre drogas. Informe 2004. Recogido el 23 de enero de 2006 en http://www.pnd.msc.es/Categoria2/publica/ pdf/oed-2004.pdf.

Plan Nacional sobre Drogas (2004a). Encuesta domiciliaria sobre el uso de drogas en España, 2003-resumen-. Recogido el 13 de Enero de 2005 en http://www.mir. es/pnd/observa/pdf/domiciliaria2003.pdf.

Plan Nacional sobre Drogas (2004b). Encuesta estatal sobre uso de drogas en enseñanzas Secundarias 2004 resumen-. Recogido el 13 de Enero de 2005 http://www. mir.es/pnd/observa/pdf/escolar2004.pdf.

Plan Nacional sobre Drogas (2004c). Encuesta sobre drogas en población escolar, 2002. Recogido el 13 de Enero de 2005 http://www.mir.es/pnd/observa/html/estudios.htm.

Sánchez-Hervás, E., Morales, E., Tomás, V., Molina, N., del Olmo, R., Bosch, C., Sanoguera, M. y Escrivá, P. (2002). Tratamiento de la dependencia a cocaína: estudio de seguimiento de seis meses. Adicciones, 14 (sup.2), 137.

San, L. y Arranz, B. (2001) Aproximaciones terapéuticas de la dependencia de la cocaína. Adicciones, 13 (sup. 2), 191-208. 


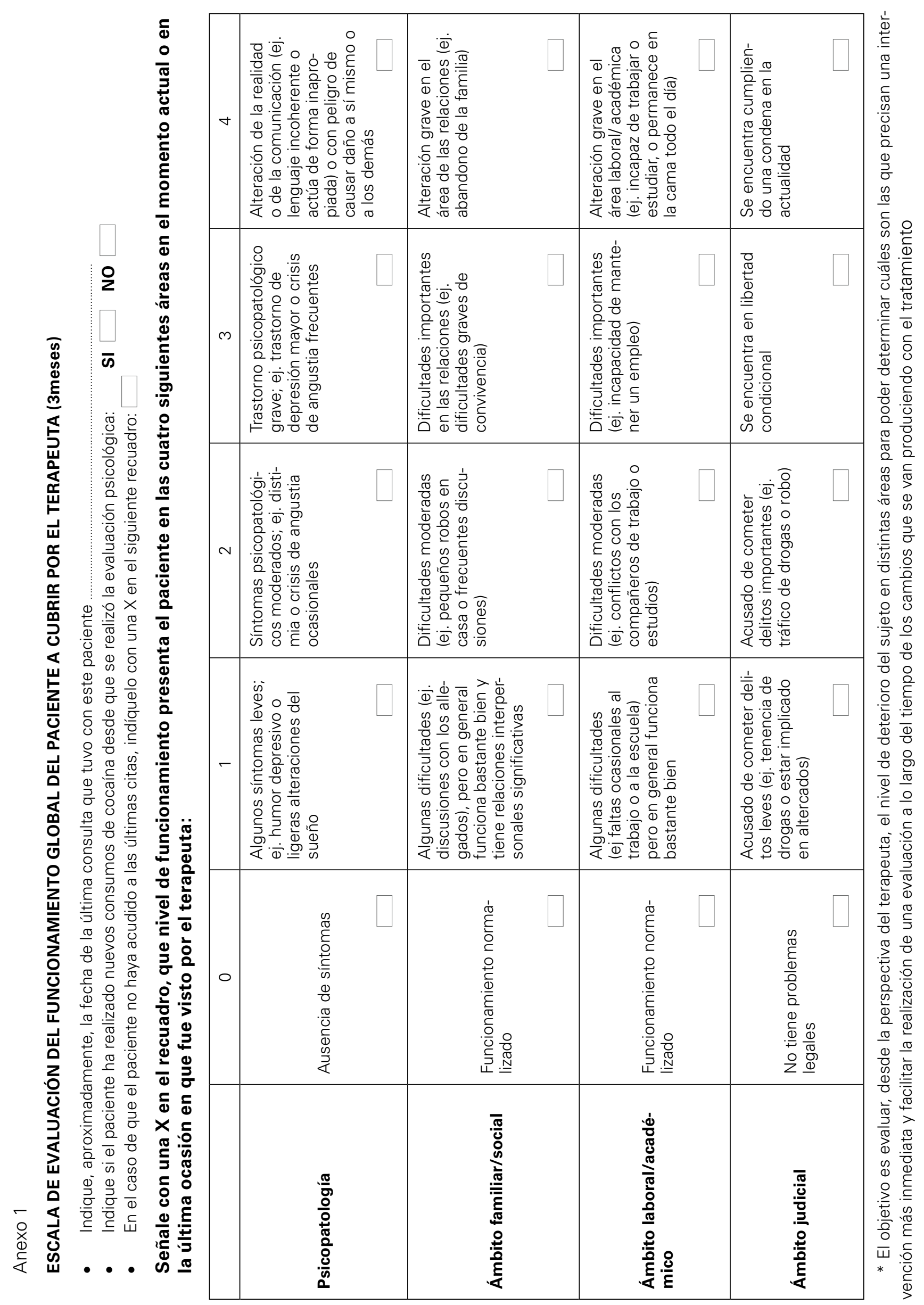


